\title{
ANTIBIOTIC SUSCEPTIBILITY PATTERNS IN POST-OPERATIVE INFECTIONS IN ORAL CANCER PATIENTS IN A TERTIARY CARE CENTRE IN SOUTH INDIA
}

\author{
Rajeevan Philip Sridhar1, Vasanth Mark Samuel2, Pranay Gaikwad ${ }^{3}$ \\ 1 1Junior Resident, Department of Surgery, Christian Medical College Hospital, Vellore, Tamilnadu, India. \\ ${ }^{2}$ Assistant Professor, Department of Surgery, Christian Medical College Hospital, Vellore, Tamilnadu, India. \\ 3 Professor and HOD, Department of Surgery, Unit-1, Christian Medical College Hospital, Vellore, Tamilnadu, India.
}

\section{ABSTRACT}

\section{BACKGROUND}

The use of antibiotic prophylaxis in head and neck oncologic surgery has greatly reduced the risk of post-operative wound infection and the corresponding increase in morbidity and health care costs.

Aim: To study the culture sensitivity pattern in patients with infections following surgical resection for oral squamous cell carcinoma and the post-operative antibiotic use in a surgical unit at a tertiary centre in South India.

\section{MATERIALS AND METHODS}

Retrospective review of case records of all patients who underwent surgical excision for oral squamous cell carcinoma under the Department of General Surgery Unit I, Christian Medical College, Vellore from January 1, 2013 to December 31, 2013.

Statistical Analysis-Epidata ${ }^{\circledR}$ was used for data entry and SPSS $20{ }^{\circledR}$ was used for data analysis.

\section{RESULTS}

A total of 86 patients underwent surgical excision of oral squamous cell carcinoma in 2013, which included 57 males and 29 females. Majority of these patients were within 41 years to 60 years of age (Range: 25 - 82 years). Thirty-five patients had carcinoma of the tongue followed by 29 with carcinoma of buccal mucosa, 17 patients with carcinoma of alveolus, 2 patients with carcinoma of lower lip, 2 patients with carcinoma of retromolar trigone and one patient with carcinoma of the floor of mouth. Thirty-three of these underwent primary closure following resection, 28 of them needed a regional flap, 14 of them underwent microvascular free-flap reconstruction and 11 needed local flaps for closure of the defect. Pseudomonas aeruginosa was the most common organism found in surgical site infections in these patients at our centre followed by other non-fermenting gram-negative bacteria and Staphylococcus aureus. Most pus cultures were polymicrobial. Pseudomonas aeruginosa was also the most common organism causing urinary tract infections in post-operative patients followed by Enterococcus and Klebsiella.

\section{CONCLUSION}

There was no statistical association between the infection rate and the site of operation or the type of operation. The surgical site infection rate was $12.8 \%$ with Cefoperazone-Sulbactam and Metronidazole antibiotic prophylaxis. The infection rate was higher when regional and microvascular free flaps were used for reconstruction of the primary defect. Since the cultures are polymicrobial with predominant gram-negative organisms, Cefoperazone-Sulbactam is a better antibiotic cover than Cefazolin.

\section{KEY WORDS}

Oral Cancer, Surgical Site Infection, Post-Operative Infection, Peri-Operative Antibiotic.

HOW TO CITE THIS ARTICLE: Sridhar RP, Samuel VM, Gaikwad P. Antibiotic susceptibility patterns in post-operative infections in oral cancer patients in a tertiary care centre in South India. J. Evolution Med. Dent. Sci. 2018;7(42):4510-4514, DOI: 10.14260/jemds/2018/1006

\section{BACKGROUND}

Infection found in the surgical site following operation of that part of the body is referred to as surgical site infection.(1) These can sometimes be superficial infections involving the skin only or more serious infections involving tissues under the skin, organs or implanted material.(2) Post-operative surgical site infections prolong hospital stay, increases cost of treatment, delays adjuvant therapy in patients with malignancies and significantly affect the morbidity of patients. (3)

'Financial or Other Competing Interest': None.

Submission 23-09-2018, Peer Review 08-10-2018,

Acceptance 08-10-2018, Published 15-10-2018.

Corresponding Author:

Vasanth Mark Samuel,

Assistant Professor, Department of Surgery,

Unit 1, Christian Medical College Hospital,

Vellore-632004, Tamilnadu, India.

E-mail: vasusanth@gmail.com

DOI: $10.14260 /$ jemds/2018/1006
Various factors determine the incidence of post-operative surgical site infection, one of them being the type of operative procedure. Operations in the head and neck region for surgical extirpation of oral squamous cell carcinoma have been classified as clean-contaminated procedures, wherein there is a constant contact of the surgical wound with oral secretions.(4) Oral squamous cell carcinoma is a major health burden in India, being the most common malignancy encountered.(5) The stage of the malignancy may also affect infection risk. Procedure-related risk factors for infection include radical or bilateral neck dissections and prolonged duration of surgery, especially if it involves reconstruction with myocutaneous flaps or microvascular free flaps.(6)

Anaerobic and aerobic bacteria are abundant in the oral cavity and oropharynx. Predominant among these include various Streptococci, Bacteroides species (excluding B. fragilis), Peptostreptococcus species, Prevotella species, Fusobacterium species, Veillonella species, Enterobacteriaceae and Staphylococci.(7) Therefore, it is not surprising that post-operative wound infections are usually polymicrobial.(7) One would expect a high post-operative 
infection rate with this array of organisms inhabiting the operative bed. Not surprisingly, post-operative wound infection rates ranging from 59 to $75 \%$ following major head and neck surgery have been reported. A double-blinded, randomised controlled study by Johnson JT showed a significant wound infection of $15 \%$ of all patients who underwent major oncologic head and neck surgery.(8) Therefore, the risk of post-operative surgical site infection has decreased with the use of antibiotic prophylaxis in head and neck oncologic surgery. This also prevents the increase in morbidity and treatment costs. Antibiotic prophylaxis has reduced the risk of severe wound infections by approximately $50 \%$. ${ }^{(9)}$ Conversely, inappropriate perioperative use of antibiotics increases costs and risk of antibiotic resistance.

Several small randomised, controlled trials significantly lower infection rates in the prophylaxis groups (5.8 - 38\%) compared with high infection rates in placebo groups (24 $78 \%$ ) using a variety of regimens including cefazolin, thirdgeneration cephalosporins and ampicillin plus Cloxacillin. Although, these were small studies, the results were concordant and the high rate of infection made the studies to attain statistical significance despite the small sample sizes. Similar results were seen in several other small, uncontrolled studies.(10-12) Antimicrobial prophylaxis targets the bacterial flora that commonly inhabit upper aerodigestive tract and the skin. It was found to be useful only in clean contaminated head and neck surgery. Broad spectrum antibiotics give the best coverage.(13)

Several randomised, single-centre studies have compared antimicrobial regimens for clean-contaminated procedures. In a study, 189 patients who were planned for head and neck cancer procedures were randomised to receive amoxicillinclavulanate $(n=97)$ or cefazolin $1 \mathrm{~g}(\mathrm{n}=92)$. In both the groups, antibiotics were given within one hour of incision and every eight hours post-operatively for three doses. This study showed an overall wound infection rate of $22 \%$. The risk of post-operative infection was more influenced by the type of surgical procedure than by disease stage. There were no significant differences between amoxicillin-clavulanate or cefazolin used as perioperative antibiotics.

Two studies have compared ampicillin-sulbactam to clindamycin and yielded discordant results. One study of 242 patients (169 evaluable) undergoing head and neck cancer procedures compared ampicillin-sulbactam $1.5 \mathrm{~g}(\mathrm{n}=119)$ and clindamycin $600 \mathrm{mg}(\mathrm{n}=123)$ given within one to two hours of incision and every six hours post-operatively for a total of four doses.(14) No difference in surgical site infection rate was found, with 15 infections reported in each group (13\% for the ampicillin-sulbactam group and $12 \%$ for the clindamycin group). There was no significant difference in adverse events between groups.

Fennessy BG et al compared ampicillin-sulbactam with clindamycin. This study with 212 patients who were planned to undergo clean-contaminated head and neck cancer surgery found lower infection rate in the ampicillin-sulbactam group $(13.3 \%)$ in comparison to the clindamycin group $(27.1 \%, \mathrm{p}=$ 0.02).(11) A greater number of gram-negative organisms were recovered from patients randomised to the clindamycin group. The combination of gentamicin and clindamycin was found to be superior to cefazolin in one of the older clinical trial.(8) The most frequently isolated pathogen in the wound infections was beta-lactamase producing Staphylococcus aureus. ${ }^{(9)}$ In a study by Becker GD et al, the most common pathogenic aerobes isolated from infected wounds were Staphylococcus aureus and beta-Streptococcus not group A, as well as a variety of Gram-negative organisms. The most common anaerobic isolate was Bacteroides melaninogenicus.(15) Gram-negative bacteria were more often isolated with Pseudomonas aeruginosa as the dominant species.

\section{MATERIALS AND METHODS \\ Type of Study}

Retrospective online chart audit. This study was reviewed and approved by the Institutional Review Board of the Christian Medical College, Vellore.

\section{Inclusion Criteria}

All patients who underwent surgical resection for oral squamous cell carcinoma under the Department of General Surgery Unit I from January 1, 2013 to December 31, 2013.

\section{Method of Collection of Data}

Retrospective online chart review.

\section{Statistical Software and Analysis}

Epidata was used for data entry and SPSS 20 was used for data analysis.

\section{RESULTS}

A total of 86 patients who underwent surgical excision of oral squamous cell carcinoma at our institute in 2013 that included 57 males and 29 females. Majority of the patients were within 41 years to 60 years of age (Range: 25 - 82 years), Figure 1 . Thirty-five patients $(41.9 \%)$ had carcinoma of the tongue followed by $29(32.6 \%)$ with carcinoma of buccal mucosa, 17 patients (19.8\%) with carcinoma of alveolus, 2 patients with carcinoma lower lip, 2 patients with carcinoma of retromolar trigone and one patient with carcinoma of floor of mouth. Thirty-three of these underwent primary closure following resection, 28 of them needed a regional flap, 14 of them underwent microvascular free flap reconstruction and 11 needed local flaps for closure of the defect.

Free flap and regional flap surgeries were most commonly associated with a wound infection rate of $21.4 \%$, Figure 2. Buccal mucosa resection surgeries and alveolar resection surgeries were most commonly associated with wound infections at $17.2 \%$ and $35.3 \%$, respectively. Infection rate in resection surgeries among other sites including tongue, lip, retromolar trigone and floor of mouth was nil.

\section{Antibiotic Administration Pattern}

The most common peri-operative prophylactic antibiotic Cefoperazone-Sulbactam and Metronidazole was received by 83 patients. 2 patients received amoxicillin-clavulanic acid and metronidazole as perioperative antibiotic, while one patient received Gentamicin and Metronidazole as perioperative antibiotic.

During the post-operative period, four patients received Piperacillin-Tazobactam and two patients received Meropenem therapeutically for infections based on cultures and antibiotic sensitivity patterns. 


\section{Surgical Site Pus Culture}

The most common organism isolated from pus cultures was pseudomonas aeruginosa followed by non-fermenting gramnegative bacteria other than Pseudomonas and Staphylococcus aureus, Table 1. Pseudomonas aeruginosa was found susceptible to Cefoperazone-Sulbactam and Piperacillin-Tazobactam. Only patients in whom surgical site infection was suspected clinically were subjected to pus culture from surgical site, Table 2 .

\section{Urine Culture}

The most common organism causing urine infection in our study was Pseudomonas aeruginosa, Enterococcus and E. coli. Pseudomonas aeruginosa was susceptible to CefoperazoneSulbactam and Enterococcus was susceptible to Linezolid, Tables 3 and 4.

\section{Sputum Culture}

There were totally 4 sputum cultures sent and one of it showed no growth. Two showed polymicrobial and the fourth sputum culture was monomicrobial. The organisms found in sputum culture were Pseudomonas aeruginosa, Enterococcus, Acinetobacter baumannii, E. coli and Klebsiella.

\section{Blood Culture}

There was no growth in any of the blood cultures sent as a part of post-operative infection workup.

A total of 16 pus cultures were sent for 13 patients with suspected surgical site infection. Eleven cultures were positive, of which 9 showed polymicrobial growth. All the patients who had growth in the pus culture had Cefoperazone-Sulbactam and Metronidazole as an intra-op antibiotic. Out of the 11 positive pus culture patients, 5 were operated for carcinoma of the buccal mucosa and 6 were operated for carcinoma of the lower alveolar. Of the 11 patients with positive pus cultures 6 had regional flap reconstructions, 3 had microvascular free flap reconstructions and 2 had primary closure of their defects.
Chi-square between infection and subsite (p-value: 0.155$)$ showed no significant association. Similarly, Chi-square between infection and type of operation (p-value: 0.478 ) did not show any significant association.

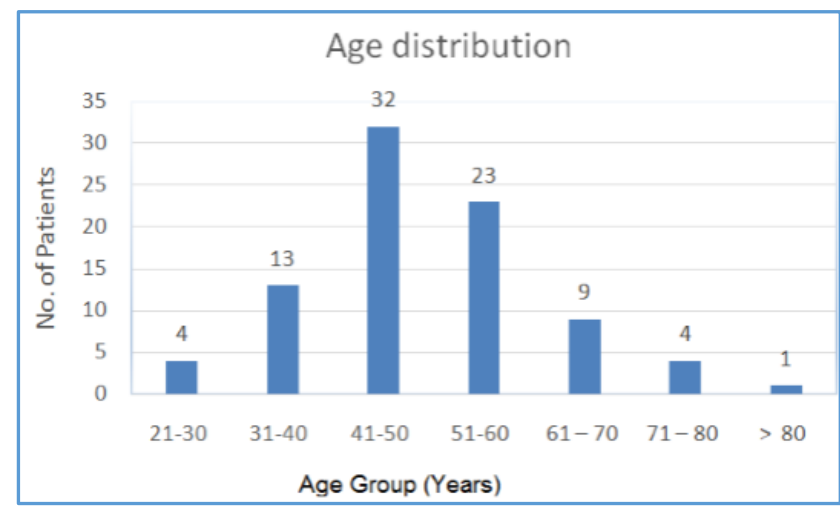

Figure 1. Age distribution of the Oral Cancer Patients operated in a Year

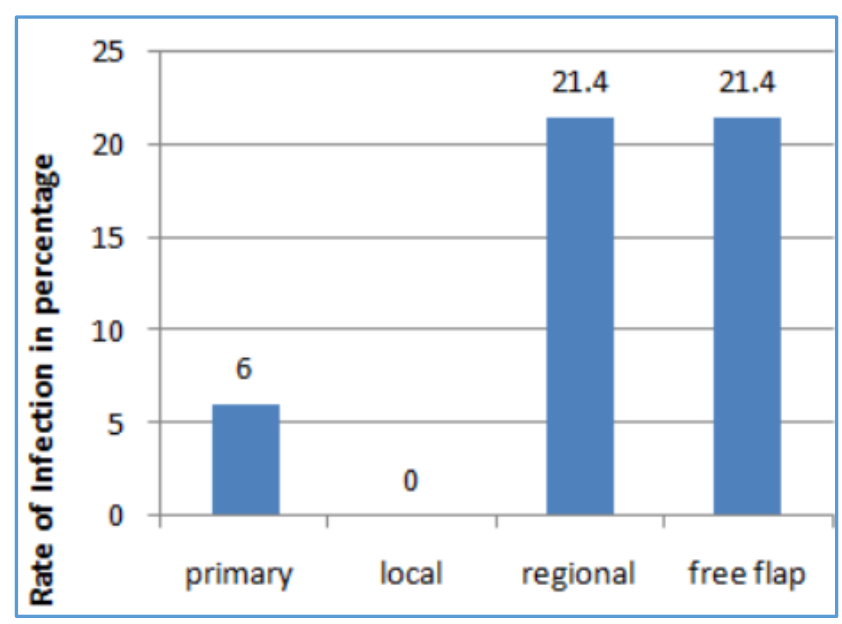

Figure 2. Infection Rate among different Types of Reconstruction

\begin{tabular}{|c|c|}
\hline Organism & Number of Cultures \\
\hline Pseudomonas aeruginosa & 6 \\
\hline Non-fermenting GNB & 5 \\
\hline Staphylococcus aureus & 5 \\
\hline Enterococcus & 3 \\
\hline Yeast & 3 \\
\hline Klebsiella & 2 \\
\hline Enterobacter & 1 \\
\hline E. coli & 1 \\
\hline Candida glabrata & 1 \\
\hline Coagulase-negative Staphylococcus & 1 \\
\hline No growth & 2 \\
\hline
\end{tabular}

Total number of pus cultures sent $=16$.

Note: The no. of cultures is not equal to no. of organisms as each culture can grow more than one organism.

\begin{tabular}{|c|c|c|c|c|c|}
\hline & $\begin{array}{c}\text { Pseudomonas } \\
\text { aeruginosa (n=6) }\end{array}$ & $\begin{array}{c}\text { Non-Fermenting } \\
\text { GNB (n=5) }\end{array}$ & $\begin{array}{c}\text { Staphylococcus } \\
\text { aureus (n=5) }\end{array}$ & $\begin{array}{c}\text { Enterococcus } \\
(\mathbf{n}=3)\end{array}$ & $\begin{array}{c}\text { Klebsiella } \\
(\mathbf{n}=2)\end{array}$ \\
\hline Antibiotic & $\begin{array}{c}\text { Susceptibility } \\
\text { Percentage }\end{array}$ & $\begin{array}{c}\text { Susceptibility } \\
\text { Percentage }\end{array}$ & $\begin{array}{c}\text { Susceptibility } \\
\text { Percentage }\end{array}$ & $\begin{array}{c}\text { Susceptibility } \\
\text { Percentage }\end{array}$ & $\begin{array}{c}\text { Susceptibility } \\
\text { Percentage }\end{array}$ \\
\hline Piperacillin & $83 \%$ & $0 \%$ & & & \\
\hline Amikacin & $50 \%$ & $0 \%$ & & & \\
\hline Ceftazidime & $83 \%$ & $0 \%$ & & & \\
\end{tabular}




\begin{tabular}{|c|c|c|c|c|c|}
\hline Cefoperazone & $66 \%$ & $40 \%$ & & & \\
\hline Levofloxacin & $50 \%$ & $0 \%$ & & $50 \%$ & $100 \%$ \\
\hline Tobramycin & $50 \%$ & $60 \%$ & & & \\
\hline Colistin & $100 \%(n=1)$ & $100 \%(n=3)$ & & & \\
\hline Imipenem & $100 \%(\mathrm{n}=1)$ & & & & \\
\hline Netilmicin & & $66 \%(n=3)$ & $100 \%(n=3)$ & & \\
\hline Oxacillin & & & $60 \%$ & & \\
\hline Rifampicin & & & $66 \%(n=4)$ & & \\
\hline Co-trimoxazole & & & $66 \%(n=4)$ & & $100 \%$ \\
\hline Linezolid & & & $100 \%(n=3)$ & $100 \%$ & \\
\hline Erythromycin & & & $60 \%$ & & \\
\hline Tetracycline & & & $100 \%$ & $0 \%$ & \\
\hline Chloramphenicol & & & $100 \%(n=3)$ & $100 \%$ & $100 \%$ \\
\hline Ampicillin & & & & $100 \%$ & \\
\hline Gentamycin & & & & $67 \%$ & $100 \%$ \\
\hline Amoxclav & & & & & $100 \%$ \\
\hline
\end{tabular}

\begin{tabular}{|c|c|}
\hline Organism & Number of Cultures \\
\hline Pseudomonas aeruginosa & 2 \\
\hline Enterococcus & 2 \\
\hline E. coli & 2 \\
\hline Klebsiella & 1 \\
\hline Candida tropicalis & 1 \\
\hline No growth & 5 \\
\hline \multicolumn{2}{|c|}{ Table 3. List of Organisms Grown in Post-Operative Urine Cultures* } \\
\hline
\end{tabular}

*Total number of urine cultures sent $=13$.

Note: The number of cultures is not equal to the number of organisms, as each culture can grow more than one organism.

\begin{tabular}{|c|c|c|c|}
\hline & Pseudomonas sp. (n=2) & Enterococcus (n=2) & E. coli (n=2) \\
\hline Antibiotic & Susceptibility Percentage & Susceptibility Percentage & Susceptibility Percentage \\
\hline Amikacin & $100 \%$ & & \\
\hline Cefoperazone & $100 \%$ & & \\
\hline Meropenem & $100 \%(\mathrm{n}=1)$ & & \\
\hline Netilmicin & $100 \%(\mathrm{n}=1)$ & & $100 \%$ \\
\hline Piperacillin & $100 \%(\mathrm{n}=1)$ & & $100 \%$ \\
\hline Imipenem & $100 \%(\mathrm{n}=1)$ & & \\
\hline Ciprofloxacin & $100 \%(\mathrm{n}=1)$ & $100 \%$ & $100 \%$ \\
\hline Linezolid & & $100 \%$ & \\
\hline Levofloxacin & & & \\
\hline Chloramphenicol & & & \\
\hline Tetracycline & & \\
\hline Co-trimoxazole & \multicolumn{2}{|l|}{} \\
\hline \multicolumn{2}{|l|}{ Table 4. Sensitivity Pattern for Urine Culture Organisms } \\
\hline
\end{tabular}

\section{DISCUSSION}

In 2013, 86 patients underwent surgical resection for oral squamous cell carcinoma. All of these patients were included in the study. The most common prophylactic antibiotic given was Cefoperazone-Sulbactam and Metronidazole, started at induction and continued for 48 hours post-operatively. In the post-operative period, patients having symptoms and signs suggestive of infection underwent a complete evaluation and workup to identify the source. The surgical site infection rate was found to be $12.8 \%$.

The most common organisms isolated in pus cultures were Pseudomonas aeruginosa (35.2\%), other nonfermenting Gram-negative Bacteria (29.4\%) and Staphylococcus aureus (29.4\%). Of the 16 patients for whom pus cultures sent, only 3 patients had a urine culture also sent as a part of fever workup. In those three patients, only one patient grew E. coli along with yeast and other two urine cultures were sterile. Of these 16 patients, only four patients had a blood culture also sent as a part of a fever workup. None of the blood cultures had growth of any significant organisms.

A total of 13 patients were suspected to have a urinary tract infection, urine cultures were sent, and the most common organism was Pseudomonas aeruginosa, Enterococcus and E. coli. Of the subsites, $17.2 \%$ of patients operated for buccal disease developed cultures with the growth of significant organisms in pus and $35.3 \%$ of patients operated for carcinoma of the alveolus developed cultures with the growth of significant organisms.

Complex reconstructive techniques using regional flaps and microvascular free flaps were associated with wound infections with pus cultures growing significant organisms in $21.4 \%$ of patients. Only $6 \%$ of patients undergoing primary closure had significant growth in pus cultures. 


\section{The Limitations of this Study were as follows-}

- $\quad$ This study was a retrospective and was conducted for a period of 1 year and so the sample size is small.

- Due to subjective differences in preparing the operation notes and discharge summaries, certain data was inadequate to be taken into audit like duration of surgery and details regarding catheterisation.

\section{CONCLUSION}

Cefoperazone-Sulbactam with Metronidazole is an effective antibiotic prophylaxis for head and neck cancer patients. The surgical site infection rate was $12.8 \%$ with CefoperazoneSulbactam and Metronidazole antibiotic prophylaxis. Antibiotic prophylaxis should be given at induction during surgery and continued post-operatively for at least 48 hours. Since the most common organism in our clinical practice at our institution is Pseudomonas aeruginosa sensitive to Cefoperazone-Sulbactam, it is an effective perioperative antibiotic cover.

\section{Recommendation}

Since the cultures are polymicrobial with predominant gramnegative organisms, Cefoperazone-Sulbactam is a better antibiotic cover than Cefazolin. The infection rate is higher in the free flap and regional flap surgeries suggesting povidoneiodine scrub preparation of parts might help in decreasing infection rates.

\section{ACKNOWLEDGEMENT}

The authors would like to acknowledge Dr Sam Marconi David, Assistant Professor, Department of Community Medicine for his assistance with data analysis and review of the manuscript.

\section{REFERENCES}

[1] Surgical Site Infection (SSI). HAI/CDC [Internet]. [Cited 2017 Feb 19]. https://www.cdc.gov/hai/ssi/ssi.html

[2] Surgical Site Infection (SSI) Event-9. pscssicurrent.pdf [Internet]. [cited 2017 May 27]. https://www.cdc.gov/nhsn/pdfs/pscmanual/9pscssic urrent.pdf

[3] Blair EA, Johnson JT, Wagner RL, et al. Cost analysis of antibiotic prophylaxis in clean head and neck surgery. Arch Otolaryngol Neck Surg 1995;121(3):269-71.

[4] Akhi MT, Ghotaslou R, Beheshtirouy S, et al. Antibiotic susceptibility pattern of aerobic and anaerobic bacteria isolated from surgical site infection of hospitalized patients. Jundishapur J Microbiol 2015;8 (7):e20309.
[5] Cancer Statistics. Cancer India | India Battles Cancer. http://cancerindia.org.in/cp/index.php/know-aboutcancer/statistics

[6] Lotfi CJ, Cavalcanti Rde C, e Silva AMC, et al. Risk factors for surgical-site infections in head and neck cancer surgery. Otolaryngol Head Neck Surg 2008;138(1):74-80.

[7] Clinical Practice Guidelines for Antimicrobial Prophylaxis in Surgery. ASHP Therapeutic Guidelines. https://www.ashp.org/-/media/assets/policyguidelines/docs/therapeutic-guidelines/therapeuticguidelines-antimicrobial-prophylaxis-surgery.ashx

[8] Johnson JT, Myers EN, Thearle PB, et al. Antimicrobial prophylaxis for contaminated head and neck surgery. Laryngoscope 1984;94(1):46-51.

[9] Friberg D, Lundberg C. Antibiotic prophylaxis in major head and neck surgery when clean-contaminated wounds are established. Scand J Infect Dis Suppl 1990;70:87-90.

[10] Sepehr A, Santos BJ, Chou C, et al. Antibiotics in head and neck surgery in the setting of malnutrition, tracheotomy, and diabetes. Laryngoscope 2009;119(3):549-53.

[11] Fennessy BG, Harney M, O’Sullivan MJ, et al. Antimicrobial prophylaxis in otorhinolaryngology /head and neck surgery. Clin Otolaryngol 2007;32(3):204-7.

[12] Seven H, Sayin I, Turgut S. Antibiotic prophylaxis in clean neck dissections. J Laryngol Otol 2004;118(3):213-6.

[13] Skitarelić N, Morović M, Manestar D. Antibiotic prophylaxis in clean-contaminated head and neck oncologic surgery. J Craniomaxillofac Surg 2007;35(1):15-20.

[14] Johnson JT, Kachman K, Wagner RL, et al. Comparison of ampicillin/sulbactam versus clindamycin in the prevention of infection in patients undergoing head and neck surgery. Head Neck 1997;19(5):367-71.

[15] Becker GD. Chemoprophylaxis for surgery of the head and neck. Ann Otol Rhinol Laryngol Suppl 1981;90(3 Pt 3):8-12. 